\title{
Impairment and Functional Interventions for Aphasia: Having it All
}

\author{
Elizabeth E. Galletta $\cdot$ A. M. Barrett
}

Published online: 4 April 2014

(C) Springer Science + Business Media New York 2014

\begin{abstract}
Aphasia, a cognitive-linguistic disorder secondary to stroke, is a frequent and often chronic consequence of stroke with detrimental effects on autonomy and health-related quality of life. Treatment of aphasia can be approached in a number of ways. Impairment-based approaches that focus on training a specific linguistic form can be implemented. Additionally, functionally oriented intervention such as supported conversation and aphasia groups are also frequently utilized when providing a treatment program for an individual with aphasia. Creating a treatment approach that includes both impairment and functional methodologies and considers how these relate to the three domains proposed by the International Classification of Functioning Disability and Health (ICF) - body functions and structure, activity, and participation-can provide an individual with aphasia an optimal treatment program that is person-centered and multi-faceted.
\end{abstract}

E. E. Galletta $(\varangle)$

Department of Speech-Language Pathology and Audiology,

Hunter College, The City University of New York, 425 East 25th

Street, P.O. Box 625, New York, NY 10010, USA

e-mail: elizabeth.galletta@gmail.com

E. E. Galletta - A. M. Barrett

Kessler Foundation Research Center, West Orange, NJ, USA

E. E. Galletta - A. M. Barrett

Department of Physical Medicine and Rehabilitation, NJ

Medical School, Rutgers-the State University of New Jersey,

Newark, NJ, USA

A. M. Barrett

Kessler Institute of Rehabilitation, West Orange, NJ, USA
Keywords Aphasia · Treatment · Intervention . International Classification of Functioning Disability and Health (ICF) - Impairment · Functional · Group therapy

\section{Introduction}

Aphasia, a cognitive-linguistic disorder that is a frequent and often chronic cognitive consequence of stroke, results in detrimental effects on autonomy and health-related quality of life. It affects approximately 1 million people in the USA today. Commonly defined as language impairment or loss, aphasia causes impairment of oral language production, language comprehension, and other associated linguistic communication skills potentially by modality (auditory, oral, visual, gestural), level of processing (phonology, morphology, syntax, semantics), or impairment in other cognitive domains relevant to functional communication (emotional communication, pragmatics, self-monitoring, theory of mind or perspective-taking, aesthetics, and humor). Regardless of the classification used, for individuals with aphasia, some or all aspects of linguistic competence are impaired.

With age as a major risk factor for ischemic stroke, and aging of the US population, aphasia will become more common in the coming decades. Current behavioral treatment strategies for aphasia, although beneficial in some cases, leave many stroke survivors with life-long disability $[1 \bullet \cdot]$. We will discuss current approaches to aphasia therapy in the context of the International Classification of Functioning Disability and Health (ICF) [2], a biopsychosocial model of health and disease that promotes the consideration of health conditions among three domains of functioning: body functions and structure, activity, and participation. See Fig. 1, below. 
Fig. 1 The international classification of functioning model (reprinted with permission from the World Health Organization) [2]
International Classification of Function Model (ICF)

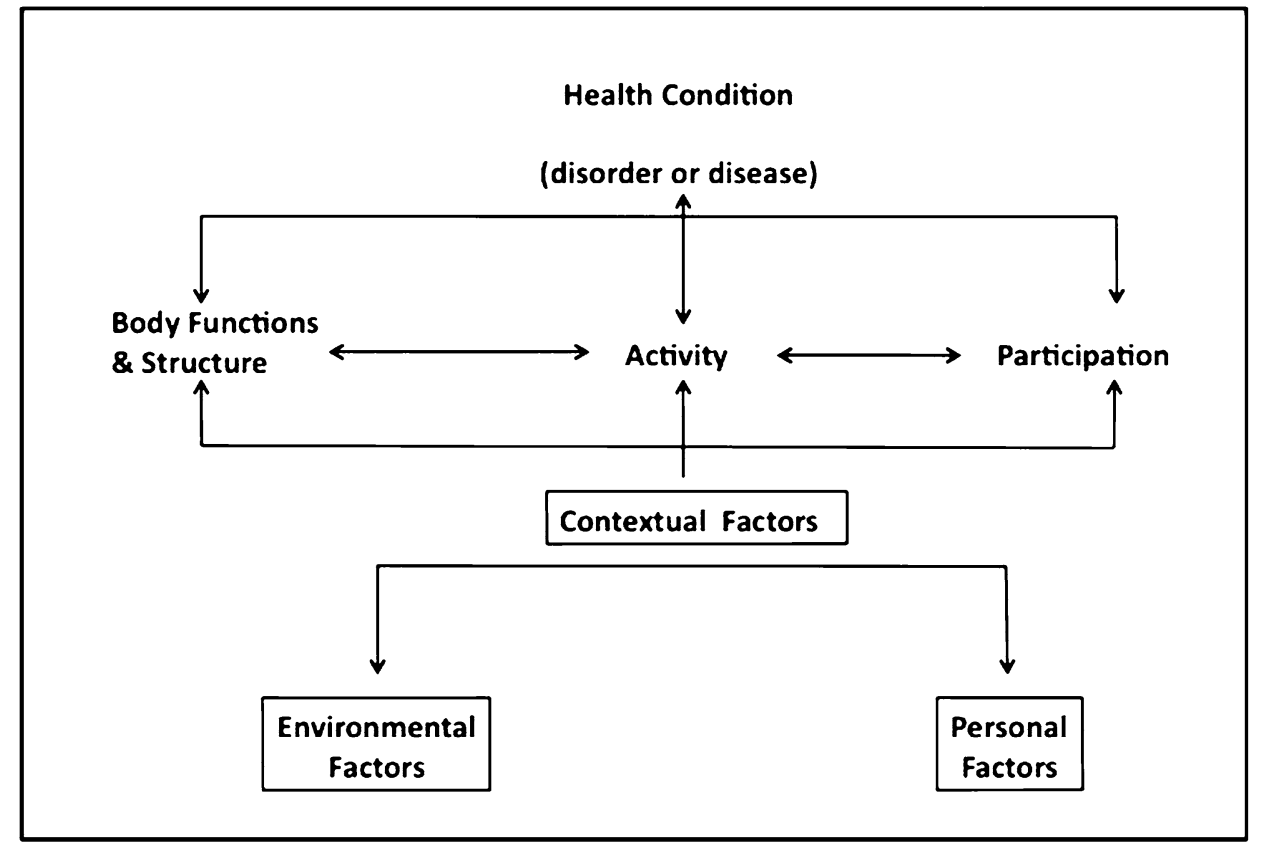

Adapted From: Model of Disability - ICF Model
In the ICF model, the "body functions and structure" domain classifies the health condition as impairment based, thus approaches to treatment that focus on the aphasia impairment reflect this domain. With regard to aphasia, there are several impairment-based approaches to behavioral intervention that focus on areas such as word finding [3] and grammar [4]. Moreover, neurophysiological clinical interventions for aphasia such as the use of noninvasive brain stimulation as an adjuvant to behavioral treatment for aphasia [5-13•] fall under the body functions and structure or the impairment-focused domain of the ICF.

The ICF domain labeled "activity" considers the life activities in which an individual engages and how the health condition affects an individual's activities. Examples of activities that may be affected secondary to aphasia are talking on the telephone, asking for directions to a location, or sharing a story. Aphasia treatment approaches that reflect this domain take into account the context and environment where communication occurs [14]. Lastly, the "participation" domain of the ICF classifies an individual's participation in society and the effects of aphasia on social roles and life situations such as attending a book club or going to a community luncheon, for example. Reflecting this domain, the life participation approach to aphasia therapy considers the conversational partner as a key part of the treatment of aphasia $[15,16]$.

There are a variety of aphasia classification systems that range from considering aphasia as a unitary disorder [17] to classifying aphasia as discretely distinct syndromes [18].
Treatment approaches for aphasia may reflect the particular classification system the clinician prefers and include, but are not limited to, "traditional" approaches, "cognitive neurolinguistic" approaches, and/or "functional" approaches to aphasia intervention. For the purposes of this article, we will discuss current trends in aphasia treatment in the context of the ICF, specifically describing approaches to aphasia intervention that are impairment-based, reflecting the body functions and structure domain, and functionally oriented, reflecting the activity and participation domains of the ICF. Research-based treatments that address the brain mechanism and neuroplasticity as well as functional reorganization of language in the brain will be discussed under the section focused on impairment-based approaches to aphasia treatment.

\section{Impairment Based Aphasia Treatment}

\section{Word Retrieval}

Word retrieval difficulty is a characteristic present in all people with aphasia regardless of the applicable aphasia classification system. Therefore, treatment of word retrieval is a common focus of intervention by nearly all clinicians implementing an impairment-based treatment approach. Before focusing on word retrieval, determining whether word retrieval difficulty reflects a semantic (meaning-based) or a phonological impairment (based on 
auditory or articulatory word forms) will help guide the treatment session. Treatment activities focused on training synonyms or antonyms, completion of fill-in the blank tasks, or tackling word description tasks are examples of activities included in a semantic approach to treatment. A specific semantic approach, referred to as semantic feature analysis (SFA) [3, 19, 20], focuses on training words with similar semantic features with the idea that overlap among features may prime or reduce random activation in the semantic system, and this might positively affect generalization. The SFA approach, in which features are generated when presented with a picture surrounded by categories of semantic features, is promoted as "semantic," yet if the clinician offers phonemic cues or rhyming cues, for example, this approach would then include both semantic and phonological training. Often, clinicians implementing a semantic approach also provide phonemic cues at some point in the session. This means that they are taking a semantic-phonological approach to treatment of word retrieval.

As noted above, treatment approaches emphasizing word sounds (for example, rhyming cues) and articulatory patterns and buccolingual movements would be considered phonological. A phonomotor approach to word-finding treatment was reported by Kendall et al. [21]. They hypothesized that training heard and produced speech sounds using various phonomotor tasks would enhance the neural connectivity supporting individual phonemes and phoneme sequences, and result in fewer phonological naming errors in individuals with aphasia. Ten people with chronic aphasia secondary to left hemisphere stroke were studied. The phonomotor treatment stimuli consisted of phonemes in both real words and non-words that were phonotactically legal in English, meaning the sound combinations were present and acceptable in English (for example in English, the sound combination /caz/ is phonotactically legal, whereas the sound combination /cza/ is not phonotactically legal). The phonomotor treatment included a total of $60 \mathrm{~h}$ of intervention (1-h sessions, two sessions per day, for 5 days a week over a treatment period of 6 weeks). The first stage of the treatment focused on sounds in isolation and the next stage included treatment of sounds in various combinations. There were two stages of treatment. Stage 1 included five tasks: exploration of sounds, motor description, perception tasks, production tasks, and graphemic tasks. Stage 2 included two additional tasks: a production and graphemic task and a perception and graphemic task. The results revealed that participants showed statistically significant improvements in accuracy on trained items on post-treatment tests, but no improvement occurred on untrained items. While this treatment description is research based, elements of this approach may be present clinically when a therapist takes an impairment-based phonemic approach to treatment of word retrieval deficits in aphasia by training certain words of high importance to the patient (for example, the name of his street) using a sound-based approach.

\section{Brain Mechanism Influence}

Although impairment-based behavioral treatments have promoted improved word production in some people with aphasia, many continue to have speech and language problems post-stroke even after they have received a course of behavioral treatment. The majority of treatments reported in the literature result in improvement on trained stimuli, but generalization to untrained material has unfortunately been limited $[1,21]$. Thus, more effective treatments are needed for the improvement of naturalistic, unconstrained speech, language, and communication post stroke. Directly considering the stage of impaired language processing and what this means about likely underlying abnormalities of brain function may help to target treatments and choose specific outcome measures to assess treatment success, and thus optimize treatment outcomes. The functional reorganization of language in people with aphasia may involve both intra- and interhemispheric interactions. Functional imaging studies have provided information on brain mechanisms during aphasia recovery. Specifically, imaging studies have indicated that activation of the language-dominant left hemisphere regions during language-related tasks has consistently been shown to have the most favorable influence on language outcomes [2225 ] and includes both the reactivation of lesioned structures and also the recruitment of additional, perilesional areas. For non-fluent aphasics, Broca's area and surrounding structures are considered the key region. In contrast, after a stroke, activation in contralesional homotopic right hemisphere regions may be upregulated. Although the mechanisms of this increase in activation are unclear, this activation may involve subcortical-cortical interactions [26] because of reduced inhibition from the left hemisphere. Researchers are still clarifying why right hemisphere activation accompanies language recovery in some people with aphasia [27-31] and under what circumstances right brain activation may be dysfunctional [33-36].

\section{Constraint-Induced Methods}

Constraint-induced language treatment (CILT) is a behavioral treatment approach for aphasia with theoretical underpinnings based on knowledge about the brain mechanism. CILT is modeled after constraint-induced movement treatment (CIMT) [37, 38], which encourages forced use of the hemiparetic hand and arm in order to promote neuroplastic changes in the lesioned hemisphere 
contralateral to the weak arm/hand, with the ultimate goal of improved movement. CILT is an intervention strategy aimed at improving the quality and quantity of verbal linguistic output of people with aphasia. The treatment focuses on reducing the reliance on compensatory (substitutive) communication strategies, such as writing and gesturing, in order to force the individual to utilize more extensive verbal means to communicate [39, 40]. It is possible that practicing oral language can promote neuroplastic changes in the left hemisphere and support improved language function. Oral verbal expression is required (and actually promoted using constrained techniques) for people with aphasia, who may previously have made extensive use of nonverbal strategies or reading/ writing to enhance communication effectiveness. The premise is that substituting other behaviors for oral verbal language may reduce the capacity for recovery of verbal linguistic neural networks; by eliminating the non-linguistic behaviors, CILT may promote left hemisphere reactivation. Using CILT, the person with aphasia is required to verbally communicate with another person, and constraints such as a barrier (blocking view of the other person's hands, anything in them, and their gestures) are used to further promote verbal descriptions. Specifically, patients may not use gestures, writing, or any other compensatory strategies; they must produce verbal oral expressive language without the support of compensatory strategies. Often people with aphasia use compensatory strategies spontaneously, and language therapy approaches over the years have promoted a compensatory approach [41, 42]. Whereas people with chronic aphasia may be in the habit of using these strategies, throughout the course of treatment using a constraint-induced approach, clinicians aim to decrease use of compensatory strategies allowing the individual to progress in terms of verbal output [40]. In addition to the focus on forced use, a main component of this technique is intensive practice. This involves utilizing an extremely time-consuming and intense treatment regimen. Patients are typically seen for treatment up to $3 \mathrm{~h}$ a day for up to 5 days a week [43].

While much of the research in the area of CILT involved people with chronic aphasia who habitually used compensatory techniques for improved communication, a smaller focus of clinical research in this area includes more recent stroke survivors. Not surprisingly, a comprehensive literature review examining the effects of this treatment for adults with aphasia [44] indicated the majority of the studies included chronic patients with aphasia. Importantly, the same review reported separate categories for CILT and intensive aphasia treatment in order to determine whether the effects of CILT were the result of the "forced use" or the "massed practice" aspects of the treatment regimen. The authors looked at ten studies and found that CILT did show some positive effects on improving language in people with aphasia. However, the authors noted these benefits were about the same as other types of intensive aphasia treatment regimens and claimed that further research must be conducted to fully understand the "constraint" piece of the treatment [44]. The major question that is still unanswered regarding CILT is whether the constrained methods, intensity of treatment, or the combination of both is what is responsible for the positive outcomes and related neuroplastic brain mechanistic changes that are proposed.

Moreover, whether the habitual use of a compensatory mechanism approach to treatment prior to CILT hinders the potential effect of CILT has not been widely studied. In a population of recent stroke survivors in which compensatory strategies had not become habitual, Kirmess and Maher [45] studied the effects of CILT in people with aphasia who resided in a rehabilitation center the first 1-3 months post stroke. The improvements in language function and patient satisfaction with this treatment were reported to be high. Not surprisingly, expressive language improvements were greater than receptive language improvements using this approach. It is possible that using CILT early on in the course of rehabilitation had a positive effect because a habitual pattern of communication post stroke had not set in. However, using a constrained approach such as CILT early on in the course of treatment post stroke may also be frustrating to recent stroke survivors. Taking into account the perspective and desires of the individual with aphasia should be considered when determining the best treatment approach to be implemented for a person with aphasia [2].

\section{Noninvasive Brain Stimulation (NBS)}

Promoting neuroplastic brain mechanism activation or reactivation of the left hemisphere is thought to be an important aspect that contributes to improved language function post stroke. Although this approach is not yet approved by the food and drug administration for clinical use, research indicates that in addition to using a behavioral method such as CILT, one way to modulate the functional reorganization of language-relevant areas in aphasia may be use of non-invasive brain stimulation (NBS). Facilitating and inhibiting stimulation protocols can be used to support optimal reactivation of the left hemisphere language networks. Stimulating the right hemisphere Broca's homolog using transcranial magnetic stimulation (TMS), which may decrease activation in this region, can support leftward lateralization and is associated with improved language recovery compared to sham stimulation in subacute stroke [46]. 
Transcranial direct current stimulation (tDCS), which, in contrast to TMS, can be conducted simultaneously with language intervention for people with aphasia, has been implemented in many aphasia rehabilitation studies. tDCS modulates cortical excitability by applying constant lowintensity electrical currents through surface electrodes on the scalp. Several studies have investigated tDCS in aphasia with promising results [5-12], although optimal methods of administration have not yet been identified. In the future, this research treatment may be used in conjunction with behavioral treatment for aphasia in standard and customary clinical treatment protocols for aphasia to accelerate recovery.

\section{Functionally Oriented Aphasia Treatment}

\section{Life Participation}

The Life Participation approach to aphasia treatment is a functionally oriented approach that focuses on improving the ability to perform communication activities of daily living [47]. This approach promotes the idea that focusing on the real-life goals of people with aphasia will allow the individual to reengage in life. The primary purpose of treatment is to reengage in communication activities that relate to real-life experiences. The treatment goals are highly specific to each individual's needs. As the person with aphasia progresses from early stages post stroke in the hospital setting to a discharge environment in a rehabilitation community, a supported-living community, or the home environment, the goals for the individual will likely change. A consumer-driven model of intervention focusing treatment on activities that make real-life differences is the theme that prevails in the life participation approach to aphasia intervention. An example of the life participation approach could involve discharge planning for a patient who is leaving the hospital. As discharge planning takes place for a new stroke survivor in an acute-care hospital, the individual with aphasia is included in the discharge planning meeting using supported conversation, and the stroke survivor with aphasia contributes to the discharge planning. In relation to the ICF model, this approach reflects both the activity and participation domains of the ICF.

\section{Supported Conversation}

This approach to conversational treatment puts the person with aphasia in the lead of the conversation, and the clinician follows the patient's lead $[16,48]$. The clinician provides language facilitation in the context of the conversation, within the natural flow of the conversational interaction. The conversational context is important for this approach, and both the clinician and the patient need to be aware of the context in order for this approach to work smoothly. Often a shared activity is the context of the conversation, allowing both conversational participants to be aware of the context. This conversational treatment approach can be used in individual treatment sessions or in the group therapy setting.

We wonder whether the supported conversation approach may provide both functional and impairmentbased support. Social conventions require code-switching, and this can be challenging for people with aphasia [49]. It is possible that the supported conversation approach reduces the need to code-switch from a comfortablefamiliar communication mode to a formal-medical mode, easing the burden of communication and increasing available lexical capacity.

\section{Aphasia Groups}

Group therapy for aphasia can theoretically take an impairment-based approach and focus on training-specific linguistic forms in the group setting or a functional approach involving the activities and participation domains of the ICF model. An aphasia group session that incorporates CILT principles into the group is an example of an impairment-focused group dynamic and reflects the body functions and structure domain of the ICF model. Although an impairment-based approach to group treatment is possible, given the nature of group dynamics and conversational interaction, functionally oriented group treatment is more widely implemented. Conversational group therapy for people with aphasia that employs a supported conversational approach to treatment is an example of a group treatment model that reflects the activity and participation domains of the ICF.

Because emotional communication enhances engagement and comprehension in aphasia $[50,51]$, we feel that incorporating activity and participation is very important to derive optimal benefit from group activities. Functionally oriented group activities will drive spontaneous expressions of feelings and group conversational exchange of socially relevant information with emotional content. This will not only help to target language that may be more relevant and useful to the individual with aphasia, but it may allow for better retention of learned material.

With advances in technology, individuals with communication disorders are increasingly using tablet technology to support aphasia rehabilitation. The iPad (Apple, Inc.) can be customized to individuals with aphasia to contain apps that are specifically relevant to a person's participation and activities [52•]. Using an iPad that has been specifically designed for an individual with aphasia as 
well as including the individual with aphasia in the selection of apps for the iPad is a treatment activity that spans both group and individual treatment modalities. Moreover, while this activity reflects the ICF activity and participation domains, training naming within a specific category using the iPad could be viewed as an impairment-based approach to treatment of naming that reflects the body functions and structure domain of the ICF. Broad implementation of the iPad in aphasia intervention could include all three domains of the ICF: body functions and structure, activity, and participation.

\section{Conclusion}

Aphasia therapy involves both impairment-based and functionally oriented approaches, as well as a system that includes individual and group treatment. The ICF [2] provides a model for classification of health conditions among three domains: body functions and structure, activity, and participation. We suggest that none of these domains is superior to the others, and an ideal program of treatment for aphasia considers each of these domains to provide the individual with aphasia the opportunity to engage in treatment at a variety of levels. Future directions in aphasia therapy are likely to continue to consider both impairmentbased and functionally oriented treatments, with the possible future use of neurophysiological approaches such as noninvasive brain stimulation (an impairment-based approach that reflects the body functions and structure domain) in conjunction with behavioral language therapy that includes training of linguistic forms in the context of conversation (ICF activity domain). Treatment that is relevant to the individual with aphasia (ICF participation domain) and treatment that includes the patient with aphasia as a partner in the treatment decision-making process should be a part of standard and customary clinical care. The ICF model provides a system that allows for classification of aphasia intervention that optimally includes both impairment-based and functionally oriented components, and acknowledges that all rehabilitation must be personcentered. The overarching goal of aphasia intervention is improvement in language and communication, and we feel that implementing impairment-based treatment as well as functionally oriented treatment, rather than treatment that reflects only one or the other domains, may provide for the best outcomes for stroke survivors with aphasia.

\section{Compliance with Ethics Guidelines}

Conflict of Interest EE Galletta declares no conflicts of interest. AM Barrett has received research Grants from the National Institutes of Health and National Institute on Disability and Rehabilitation Research.

Human and Animal Rights and Informed Consent All studies by AM Barrett involving animal and/or human subjects were performed after approval by the appropriate institutional review boards. When required, written informed consent was obtained from all participants.

\section{References}

Papers of particular interest, published recently, have been highlighted as:

- Of importance

•• Of major importance

1. • Raymer A, Beeson P, Holland A, et al. Translational research in aphasia: from neuroscience to neurorehabilitation. J Speech Lang Hear Res. 2008;51(Suppl):S259-S275.

2. World Health Organization. ICF: International Classification of Functioning, Disability, and Health. Geneva, 2001.

3. Boyle M. Semantic feature analysis treatment for anomia in two fluent aphasia syndromes. Am J Speech Lang Pathol. 2004;13:236-49.

4. Thompson CK, Shapiro L. A linguistic ap- proach to treatment of agrammatic aphasia. Aphasiology. 2005;19:1021-36.

5. Baker JM, Rorden C, Fridriksson J. Using transcranial directcurrent stimulation to treat stroke patients with aphasia. Stroke. 2010;41(6):1229-36.

6. Fiori V, Coccia M, Marinelli CV, et al. Transcranial direct current stimulation improves word retrieval in healthy and nonfluent aphasic subjects. J Cogn Neurosci. 2011;23(9):2309-23.

7. Flöel A, Meinzer M, Kirstein R, et al. Short-term anomia training and electrical brain stimulation. Stroke. 2011;42(7):2065-7.

8. Fridriksson J, Richardson JD, Baker JM, et al. Transcranial direct current stimulation improves naming reaction time in fluent aphasia: a double-blind, sham-controlled study. Stroke. 2011;42(3):819-21.

9. Kang EK, Kim YK, Sohn HM, et al. Improved picture naming in aphasia patients treated with cathodal tDCS to inhibit the right Broca's homologue area. Restor Neurol Neurosci. 2011;29: $141-52$.

10. Marangolo P, Marinelli CV, Bonifazi S, et al. Electrical stimulation over the left inferior frontal gyrus (IFG) determines longterm effects in the recovery of speech apraxia in three chronic aphasics. Behav Brain Res. 2011;225(2):498-504.

11. You DS, Kim DY, Chun MH, et al. Cathodal transcranial direct current stimulation of the right Wernicke's area improves comprehension in subacute stroke patients. Brain Lang. 2011;119(1):1-5.

12. Marangolo P, Fiori V, Calpagnano M, et al. tDCS over the left inferior frontal cortex improves speech production in aphasia. Front Hum Neurosci. 2013;7:1-9.

13. - Hamilton RH, Chrysikou, EG, Coslett B. Mechanisms of aphasia recovery after stroke and the role of noninvasive brain stimulation. Brain and Lang. 2013: 118(1-2):40-50.

14. Lubinsky R. Environmental approach to adult aphasia. In R. Chapey (Ed.), Language intervention strategies in adult aphasia. 5th ed. Baltimore: Lippincott, Williams \& Wilkins, Philadelphia, 2008:319-348.

15. Kagan A. Supported conversation for adults with aphasia: methods and resources for training conversation partners. Aphasiology. 1998;12:851-64.

16. Simmons-Mackie N. In support of supported communication for adults with aphasia: clinical forum. Aphasiolgy. 1998;12:831-8. 
17. Schuell H, Jenkins JJ, Jiménez-Pabón E. Aphasia in adults. New York: Harper and Row; 1964.

18. Goodglass H, Kaplan H, Barresi B. Boston diagnostic aphasia examination-third edition (BDAE-3). Lea and Febiger, Philadelphia, 2001.

19. Boyle M, Coelho CA. Application of semantic feature analysis as a treatment for aphasic dysnomia. Am J Speech Lang Pathol. 1995;4:94-8.

20. Boyle M. Semantic feature analysis treatment for aphasic word retrieval impairments: What's in a name? Top Stroke Rehabil. 2010;17(6):411-22.

21. Kendall DL, Pompon RH, Brookshire CE, et al. An analysis of aphasic naming errors as an indicator of improved linguistic processing following phonomotor treatment. Am J Speech Lang Pathol. 2013;22(2):S240-9.

22. Fridriksson J. Preservation and modulation of specific left hemisphere regions is vital for treated recovery from anomia in stroke. J Neurosci. 2010;30(35):11558-64.

23. Heiss WD, Kessler J, Thiel A, et al. Differential capacity of left and right hemispheric areas for compensation of poststroke aphasia. Ann Neurol. 1999;45(4):430-8.

24. Warburton E, Price CJ, Swinburn K, et al. Mechanisms of recovery from aphasia: evidence from positron emission tomography studies. J Neurol Neurosurg. Psychiatr. 1999;66(2):155-61.

25. Meinzer M, Flaisch $T$, Breitenstein $C$, et al. Functional rerecruitment of dysfunctional brain areas predicts language recovery in chronic aphasia. Neuroimage. 2008;39(4):2038-46.

26. Crosson B, Moore AB, Gopinath $\mathrm{K}$, et al. Role of the right and left hemispheres in recovery of function during treatment of intention in aphasia. J Cogn Neurosci. 2005;17(3):392-406.

27. Thiel A, Herholz K, Koyuncu A, et al. Plasticity of language networks in patients with brain tumors: a positron emission tomography activation study. Ann Neurol. 2001;50(5):620-9.

28. Thiel A, Habedank B, Herholz K, et al. From the left to the right: how the brain compensates progressive loss of language function. Brain Lang. 2006;98(1):57-65.

29. Fridriksson J, Morrow-Odom L, Moser D, et al. Neural recruitment associated with anomia treatment in aphasia. Neuroimage. 2006;32(3):1402-12.

30. Fridriksson J, Moser D, Bonilha I, et al. Neural correlates of phonological and semantic-based anomia treatment in aphasia. Neuropsychologia. 2007;45(8):1812-22.

31. Cherney LR, Small SL. Task-dependent changes in brain activiation following therapy for nonfluent aphasia: discussion of two individual cases. J Int Neuropsychol Soc. 2006;12(6):828-42.

32. Belin P, Van Eeckhout P, Zilbovicius M, et al. Recovery from nonfluent aphasia after melodic intonation therapy: a PET study. Neurology. 1996;47(6):1504-11.

33. Rosen HJ, Petersen SE, Linenweber MR, et al. Neural correlates of recovery from aphasia after damage to left inferior frontal cortex. Neurology. 2000;55(12):1883-94.

34. Naeser MA, Martin PI, Nicholas M, et al. Improved picture naming in chronic aphasia after TMS to part of right Broca's area: an open-protocol study. Brain Lang. 2005;93(1):95-105.

35. Winhuisen L, Thiel A, Schumacher B, et al. Role of the contralateral inferior frontal gyrus in recovery of language function in poststroke aphasia: a combined repetitive transcranial magnetic stimulation and positron emission tomography study. Stroke. 2005;36(8):1759-63.

36. Heiss WD, Thiel A. A proposed regional hierarchy in recovery of post-stroke aphasia. Brain Lang. 2006;98(1):118-23.

37. Taub E, Morris DM. Constraint-induced movement therapy to enhance recovery after stroke. Curr Atheroscler Rep. 2001;3(4): 279-86.

38. Meinzer M, Elbert T, Djundja D, et al. Extending the constraintinduced momvement therapy (CIMT) approach to cognitive functions: constraint-induced aphasia therapy (CIAT) of chronic aphasia. J Neurol Rehabil. 2007;22:311-8.

39. Pulvermuller F, Neininger B, Elbert T, et al. Constraint-induced therapy for chronic aphasia after stroke. Stroke. 2001;32:1621-6.

40. Raymer A. Constraint-induced language therapy: a systematic review. The ASHA Leader, New York, 2009.

41. Ylvisaker, M., Szekeres, S.F., Feeney, T. Communication disorders associated with traumatic brain injury. In: Chapey R, ed. Language intervention strategies in aphasia and related neurogenic communication disorders. 5th ed. Baltimore: Lippincott, Williams \& Wilkins; 2008: 915-918.

42. Carney N, Chesnut RM, Maynard H, et al. Effect of cognitive rehabilitation on outcomes for persons with traumatic brain injury: a systematic review. J Head Trauma Rehabil. 1999;14:277-307.

43. Faroqi-Shah Y, Virion CR. Constraint-induced language therapy for agrammatism: role of grammaticality constraints. Aphasiology. 2009;23(7/8):977-88.

44. Cherney LR, Patterson JP, Raymer A, et al. Evidence-based systematic review: effects of intensity of treatment and constraint-induced language therapy for individuals with strokeinduced aphasia. J Speech Lang Hear Res. 2008;51:1282-99.

45. Kirmess M, Maher LM. Constraint induced language therapy in early aphasia rehabilitation. Aphasiology. 2010;24(6-8):725-36.

46. Weiduschat N, Thiel A, Rubi-Fessen I, et al. Effects of repetitive transcranial magnetic stimulation in aphasic stroke: a randomized controlled pilot study. Stroke. 2011;42(2):409-15.

47. Chapey R, Duchan JF, Elman RJ, et al. Life-participation approach to aphasia: a statement of values for the future. In: Chapey R, ed. Language intervention strategies in aphasia and related neurogenic communication disorders. 5th ed. Baltimore: Lippincott, Williams \& Wilkins; 2008: 279-289.

48. Damico J. Whole language for special needs children. New York: Buffalo: Educom Associates; 1992.

49. Fabbo F, Skrap M, Aglioti S. Pathological switching between languages after frontal lesions in a bilingual patients. J Neurol Neurosurg Psychiatr. 2000;68(5):650-2.

50. Reuterskiold C. The effects of emotionality on auditory comprehension in aphasia. Cortex. 1991;27(4):595-604.

51. Barrett AM, Crucian GP, Raymer AM, et al. Spared comprehension of emotional prosody in a patient with global aphasia. Neuropsychiatr, Neuropsychol, Behav Neurol. 1999;12(2):117-20.

52. - Hoover EL, Carney A. Integrating the iPad into an intensive, comprehensive aphasia program. Semin Speech Lang. 2014;35(1): $25-37$. 